Proceedings of the Second Annual Forestry Symposium 1996: Management and Sustainable Utilization of Forest Resources, Sri Lanka, 6-7 December 1996. (Eds. Amarasekera, HS, Ranasinghe, D M S HK and Finlayson, $W$. Published by Department of Forestry and Environmental Science, University of Sri Jayewardenepura, Sri Lanka (1998)

\title{
BIODIVERSITY IN THE CATCHMENT OF HIYARE RESERVOIR
}

\author{
M.P. de Silva \\ Department of Botany. University of Ruhuna
}

\begin{abstract}
Data are presented on the site characteristics and land-use types, and on species of trees, shrubs, fishes, tetrapod reptiles, snakes, amphibians and birds. The endemicity of the tree and shrub species is especially high, at +7.5 and $31.5 \%$ The catchment is therefore important for the preservation of biodiversity, as well as for its contribution to the water supply of the town of Calle.
\end{abstract}

\section{Introduction}

Hiyare is a natural reservoir with an area of 22.22 ha, in Galle District, in the south-east of Sri Lanka (Fig. 1). It is located at about $6^{\circ} 3^{\prime} \mathrm{N}, 80^{\circ} 21^{\prime} \mathrm{E}$, and $100 \mathrm{~m}$ above sea level. Galle District falls within the low-country wet zonc. with a moderately high mean annual rainfall of more than $2500 \mathrm{~mm}$. The district is in the agro-ecological region WLt (determined primarily by rainfall and elevation). The 75\% expectancy rainfall for this region is more than $1525 \mathrm{~mm}$ (i.c. this is the least amount of rainfall cxpected, 3 years out of 4). The area around the reservoir is characterized by red-yellow podzolic soils with soft or hard laterite and bog or semi-bog conditions. The land is flat or undulating. with occasional rolling hilly terrain.

The reservoir is an important source of pure drinking water for the people of Galle, capital of the Southern Province. The water supply system was commissioned in 1911. It provides $1.8 \times 10^{6}$ litres daily, which is a fifth of the requirements of the lown, through a $15-\mathrm{km}$ pipeline. The rest of the supply is taken from the River Gin. A well preserved lowland rain forest, the Hiyare Forest Reserve, with an area of about 250 ha, occupies part of the catchment, which also includes some scrub forests, grasslands and paddy fields (Fig. 2). The Mahadola, a tributary of the Gin, rises in the western part of the catchment. The preservation of this catchment is therefore very important. This study was under-taken to investigate its biodiversity, in order to provide some useful basic data.

\section{Methodology}

The flora was identified along a number of line transects across the catchment. A list of fauna was compiled from the observations of the survey team, supplemented by interviews with the forest officers and the local people. The land-use pattern was determined from aerial photographs. 


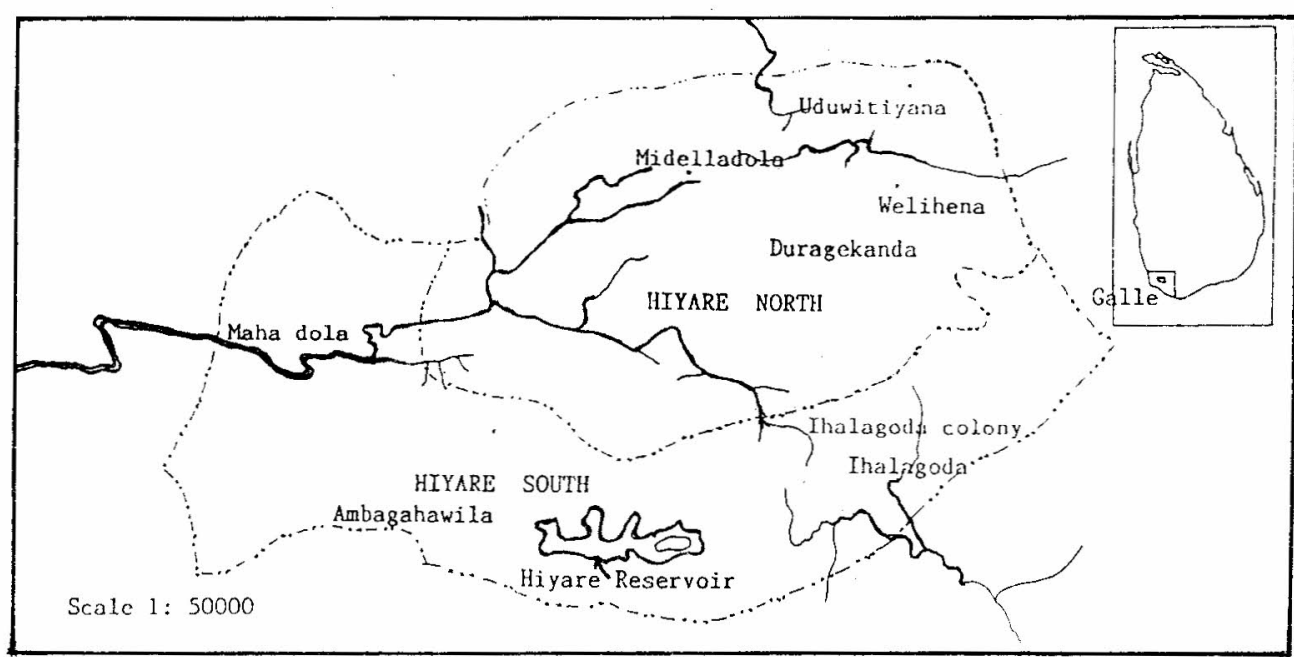

Figure 1:Location of the Hiyare Reservoir and the catchment in the Gall District

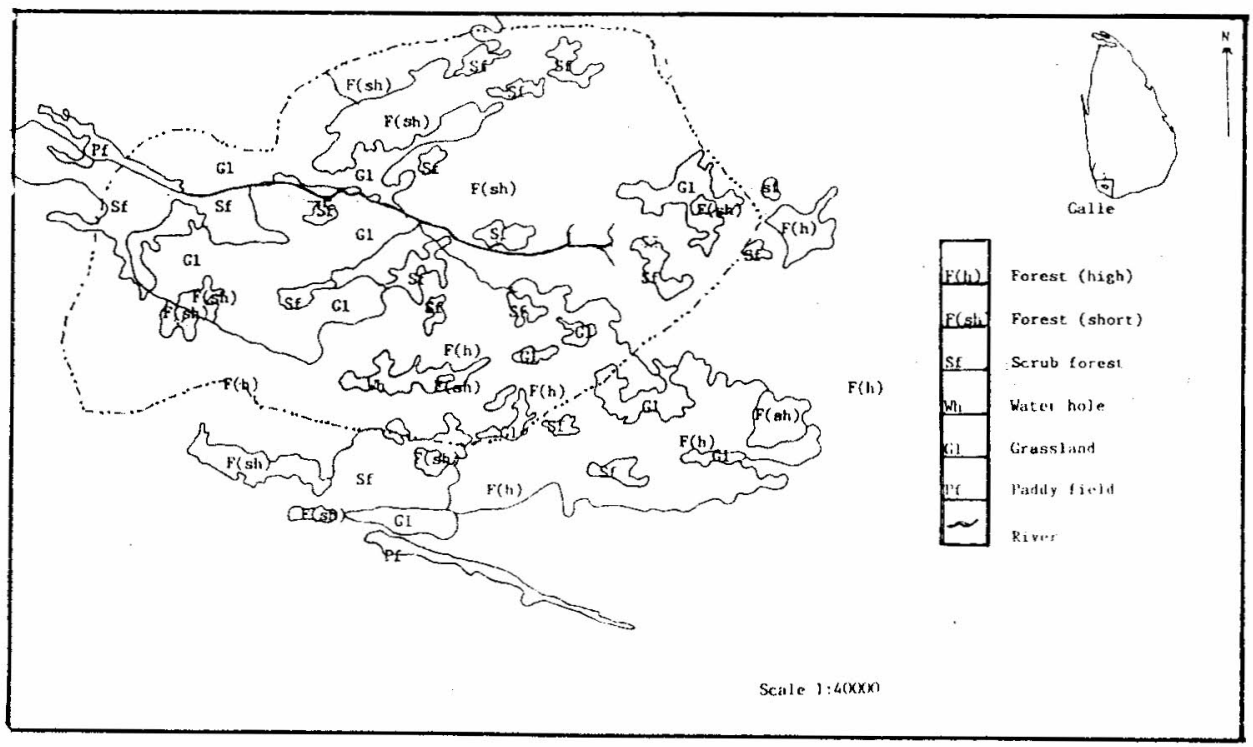

Figure 2: Landuse pattern in the Hiyare Forest Reserve

\section{8}




\section{Results and discussion}

Fig. 3 illustrates the average monthly rainfall during the present decade (data from Hiyare rain gauge). April to July $(1020 \mathrm{~mm})$ and September to November $(790 \mathrm{~mm})$ are the main rainy seasons, while generally dry conditions prevail during January to March and in August, although there is some rain throughout the year.

According to aerial photograph interpretations, the catchment comprised the following land-cover types, by percentages of the total land area:

$\begin{array}{lr}\text { Forests with taller trees }(>15 \mathrm{~m}) & 19 \\ \text { Forests with shorter trees }(<15 \mathrm{~m}) & 30 \\ \text { Shrub forest } & 14 \\ \text { Grassland } & 32 \\ \text { Water-fed areas } & 2 \\ \text { Paddy fields } & 3\end{array}$

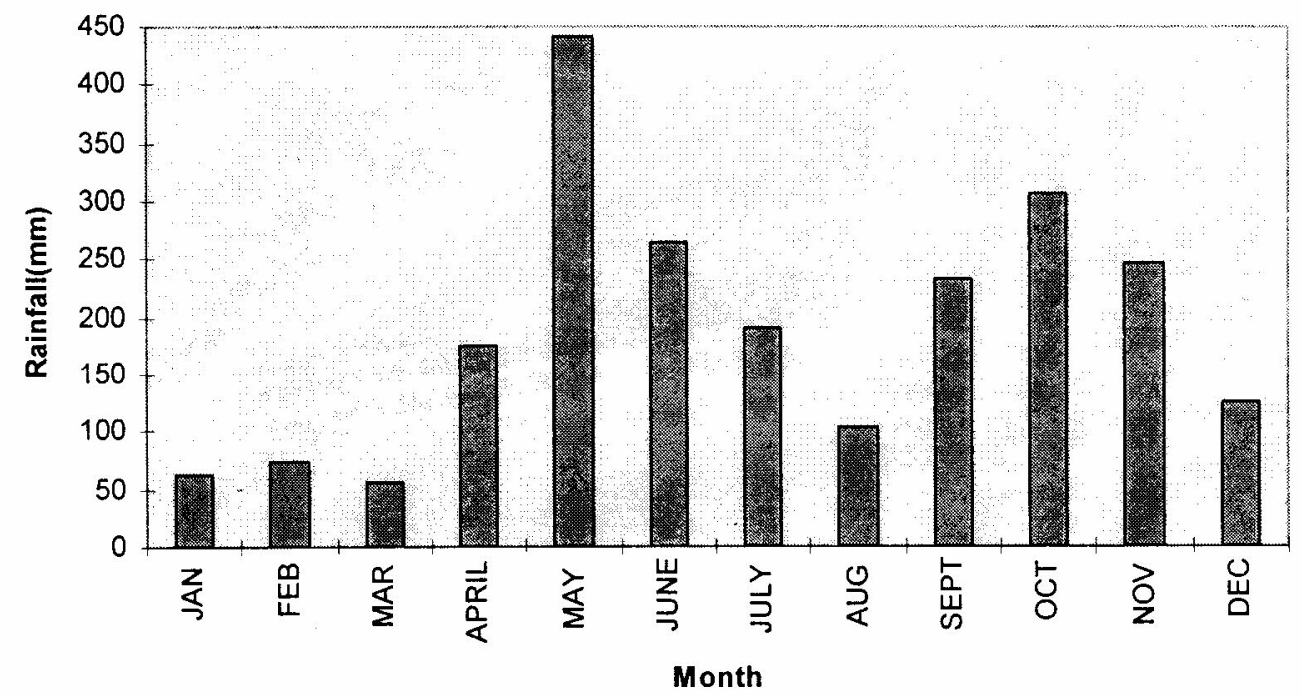

Figure 3 : Average rainfall at Hiyare (mm) 1990-96

The vegetation showed a very high percentage of endemicity. Of the 40 tree and 19 shrub species identified, $19(47.5 \%)$ and $6(31.5 \%)$ were endemic, respectively.

In the appendices, endemics are marked with an asterisk. Appendices I and 2 list the trees and shrubs identified. Notable canopy trees include Dipterocarpus glandulosus (dorana), D. hispidus (bu-hora), D. congestifolia, Canarium zeylanicum (kekuna), Chaetocarpus coriaceus (hedawaka), Kurrimia ceylanica (uruhonda), Calophyllum moonii (domba kina), and Palaquium grande (kirihembiliya) - these vernacular names, and the ones given below, are Sinhala. 
Fig. 4 illustrates the distribution of flora and fauna within the catchment. The number of endemic species encountered within each group is also given. Appendices 3 to 7 list the fresh-water fishes, tetrapod reptiles, snakes, amphibians and birds that were identified. Notable among the fresh water fishes in the streams, especially in the Mahadola, are Puntius nigrofasciatus (bulath hapaya), Rasbora vaterifloris (halmaldandiya), and Horadandia athukorali (hora dandiya). Among the birds, Galloperdrix bicalacarata (lanka haban kukula) and Ocyceros gingalensis (Sri Lankan gray hornbill) can still be seen in this environment.

From these data, Hiyare can be classified as an area of rich biodiversity. Its preservation is therefore of two-fold significance, first because of its biodiversity and second because of its water resources.

\section{Acknowledgements}

The author wishes to acknowledge the assistance of G.G. Gammachi (Forest Officer, Hiyare), R. Karunatilake of the Department of Botany, University of Ruhuna, and of Madura de Silva, A.K. Wijesekera and Janaka Gamachi of the Young Zoologist's Association of Galle.

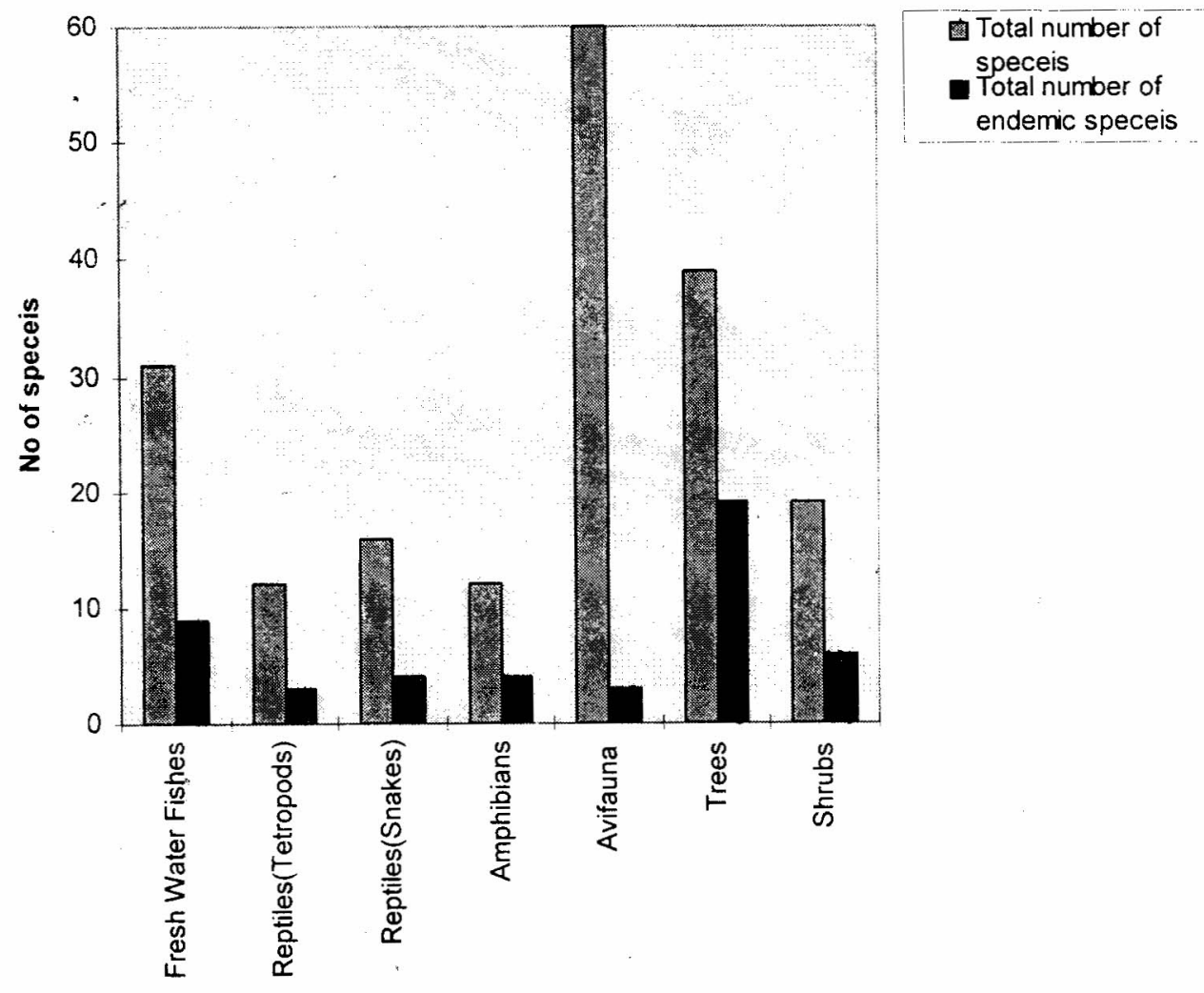

Figure 4 : Distribution of flora and fauna (total number of species and number of endemics identified) 


\section{Appendix 1 - Tree species}

Rhizophoraceac

Anisophyllea cinnamomoides*

Carallia brachiata

Celastraceac

Kurrimia ceylanica*

Myristicaceac

Horsfieldia invaghedi*

M.Mristica dactyloides

Fabaceac

Pericopsis mooniana

Erythrina variegata

Sapindaceac

Filicium decipiens

Clusiaceac

Garcinia quaesita*

Calophyllum moonii*

Calophyllum cordato-oblongum*

Menispermaceae

Cyclea burmami

Dipterocarpaceac

Dipterocarpus glandulosus*

Dipterocarpus hispidus*

Doona congestifolia*

Sapotaceac

Palaquium grande*

Dilleniaceace

Dillenia retusa*

Wormia triquetra*

Moraceac

Arfocarpus nobilis*

Burseraceac

Canarium zeylanicum*

Lecythidaceac

Careya arborea

Apocynaceae

Alstoria scholaris

Alstonia macrophylla

Myrtaceac

Syzygium carvophyllatum

Syzyguim rubicundum

Palmae

Caryota urens

Calamus delicatulus*

Calamus rotong

Anacardiaceac

Mangifera zeylanica*

Lammea coromandelica

Semecarpus gardneri* welipema

dawata

uruhonda

ruk

malaboda

nadun

erabadu

pehimbiya

ratagoraka

domba kina

kalu kina

kehipittan

dorana

buhora

tiniya

kirihembiliya

godapara

diyapara

bedi-del

kekuna

kahata

ruk-attana

havari nuga

dan

pinibaru

kitul

nara-wel

we-wel

etamba

hik

badulla 
Verbenaceac

Vinex allissima

Euphorbiaceac

Bridelia retusa

Macaranga pellata

Aporosa cardiosperma*

("haetocarpus coriaceus*

Cornaceac

Mastixia tetranda var. thwaitesii

Rubiaceac

Wendlandia bicuspidala

Menispermaceac

Cissampelos pareira milla

keta-kala

kenda

kampotta

liedawaka

diya-mitta
Melastomataceae

Osbeckia aspera

Osbeckia ockandra

Hippocrataceac

Salacia reticulata

Apocynaceac

Pagiamtha dichotonta

Araceac

Pothos scandens

Thymclaeaceac

Ciyrinops walla

Euphorbiaceae

Aporosa lindleyana

Dilleniaceac

Tetracera samentosa

Schumacheria castamearfolia*

Fabaceac

Encada puscietha

Pongamia pimala

Flagellariaceae

Flagellaria indica

Lauraceac

Neolissea cassia*

Rhamnaceace

Lyphins napeca*

Piperaceac

Piper sylvestre

Liliaceae

Asparagus falcatus

Ochnaceac

Oclma jabotopita*

Bambusaceae

Ochlomdra stridula*

Ancistrocladaceac

Ancistrocladis namatus*
Appendix 2 - Shrubs

bowitiya

heen-bowitiya

himbutu-wel

divi-kaduru

pola-wel

patta-ivala

kebella

korossi-wel

kekiri-wel

pus-wel

magul karanda

goyi-wel

dawul-kurunda

yak-eraminiya

wal-gammiris wel

hatawariya

mal-kera

bota

gona-wel 
Appendix 3 - Fresh water fishes

$\left(^{+}=\right.$introduced $)$

Anguilidae

Anguila bicolor

Cyprinidae

Damio malabaricus

Esomus thermoicos

Horadandia athukorali*

P'untius tittera*

Puntius bimaculatus*

Pumtius dorsalis

Pumtius filamentosus

P'untius nigrofasciants:

P'untius vittalus

Rasbora caverii

Rashora damiconius

Rashora varifloris*

Coboitidae

Lepidocephalichthvs thermalis

Balitoridac

Schistura notostigna

Siluridae

(Impok bimaculalus

Clariidae

(larias brachysoma*

Heteropheustidae

Heterophenstes fossilis

Aplocheilidae

Aplocheilus wemeri*

Cichlidae

Etroplus suratensis

Saratherodon mossambicus

Orechronis miloticus ${ }^{+}$

Gobiidae

Awours grammepomus

Anabantidac

Anabus testudinens

Belontidac

Belontia signata*

Osphronemidac

() sphronemus gorami ${ }^{+}$

Channidac

('hamma gachua

( 'hamna marulius

('hamma orientalis*

Chamma striata

Mastacembelidac

Mastacembelus armatus

level-finned eel

giant danio

llying barb

horadandia

cherry barb

red-side barb

long-snouted barb

tillamented barb

black-ruby barb

silver barb

striped rasbora

golden rasbora

common spiny loach

banded mountain loach

buller cattish

walking cattish

stinging cattish

Wemer's killifish

pearl spot

tilapia

tilapia

scribbled goby

climbing perch

combtail

giant gourami

brown snakchead

giant snakchead

smooth-breasted snakehead

murrel

marbled spiny eel walapoththa

kalu aandha

rath kailaya

ravul dandia

hora dandia

letiteya

ipilli kadaya

katu kureya

pethiya

bulath hapaya

podi pethiya

dandiya

hal mal dandiya

ehirava

hunga

iri handeya

koraliya

tilapia

tilapia

weligouva

kavaiya

thalkossa

seppali

parandal kanaya

ara

kola kanaya

loola

gan theliya 
Appendix $\&$ - Tetrapod reptiles

Emydidae

Melamochelys trijuga

hard-shelled terrapin

gal ibba

Trionychidac

Lissemys puinctala

Gekkonidae

soft-shelled terrapin

kiri ibba

Cinemaspis tropidogaster

Gehvera murilata

Hemidactylus depressus

Hemidactylus frenatus

Agamidae

Calotes calotes

Calotes liolepis*

Calotes versicolor.

Ceratophora aspera*

Lyriocephalus scutatus*

Otocryptis wiegmami*

Varanidae

Varamms bengalensis

Varamis salvator.

white house gecko

jungle gecko

house gecko

green garden lizard

common agamid lizard

rough-nose horned lizard

hum-nosed lizard

earless lizard

land monitor

walter monitor sudu geval huna

hali huna

geval huna

pala katussa

gara katussa

gata ang-katussa

karamalbodilima

pinum katussa

thalagoya

kabaragoya

\section{Appendix 5 - Snakes}

\section{Uropeltidae}

Cylindrophis maculatus*

\section{Boidae}

Python moltmws

Colubridae

Elaphe helena

Lycodon aulicus

Macropysthodon plumb color

Cercaspis curimatus*

()ligodon toeniolatus

Boiga cevlonemsis

Dendrelaphis Iristis

Dendrelaphis bifrenalis

Ahaetulla nasutus

Jenochrophis asperrimus*

\section{Elapidae}

Naja naja

Viperidae

Vipera russelli

Hypnale hypuale*

Trimeresurus rigonocephalus*

pipe snak
python
trinket snake
common wolf snake
green keelback
Sri Lankan wolf snake
variegated kukri snake
Sri Lanka cal snake
Seba's bronze-back
Boulenger's bronze-back
green whip snake
Sri Lanka checkered diya
kecl-back
cobra
Russel's viper
Merrem's hump-nosed vip
green pit viper

depath naya

pimbura

kata kaluwa

alu radanakaya

wal gandiya

dara karawala

wairidathkatiya

nidi mapila

tura haldanda

panaduru-

haldanda

ahatulla

naya, polonga

naya

tith polanga

kunakatuwa

pala polanga 


\section{Appendix 6 - Amphibians}

Bufonidae

Bufo atukerali*

Bufo A Relomostictus

Microhylidae

Saloula pulchra

Rhacophoridae

Philaustus variabilis

Polypedates eques*

Rhacophorus microlymipamum"

Atukorale's dwart toad

common toad

red-banded kaloula

variable bush trog montane hourglass treetish kandukara gas gemba

\section{Ranidac}

Rema anrantiaca

Rana corrugala*

Rama crassa

Rana cyanophlyctis

Rama hexadactyla

Rama limurocharis small-eared tree frog

lesser wood trog Sri Lanka corrugated frog kudu haile madiya water skipper six-loed lrog Indian reed frog atukoralage miti gemba goda gemba

rathu vichithra gemba

vichithra panduru gemba

kudukin

ruk gemba

kadawana madiya lanka ralli madiya

atikiththa

kola madiya

indiyanu pan madiya

Appendix 7 - Birds

( $m=$ migrant; $m r=$ migrant $/$ resident $)$

\section{GALLIFORMES}

Phasianidae

Galloperdix bicalcarala*

kukula

Sri Lanka spurfowl

lanka haban

PICIFORMI:S

Picidae

Dinopium ben-gholense

('luysecolcoptes heidus

Megalaimidac Captionidac

Megalaimidae zeylanica

Megalaima rubricapilla

BUCEROTTFORMLS

Bucerotidae

Ocveeros gingalensis*

\section{CORACIIFORMES}

Coraciidae

Coracias bengalensis

Alcedenidac

Alcedo athis.s

Alcedo meninting

Dacelonidace

Pelargopsis capensis

Halcyon snvmensis

Meropidae

Merops philippimus

red-backed woodpecker

crimson-backed woodpecker

brown-headed barbet

Sri Lanka small barbet

Sri Lanka gray hombill

Indian roller

common kinglisher

blue-eared kinglisher

stork-billed kingfisher

white-breasted kingtisher

blue-tailed bee-eater 


\begin{tabular}{|c|c|}
\hline \multicolumn{2}{|l|}{ CUCULIFORMES } \\
\hline \multicolumn{2}{|l|}{ Cuculidac } \\
\hline C'uculus canorus" & common cuckoo \\
\hline Eduynamys scolopacea & kocl \\
\hline Cembopus simensis & common coucal \\
\hline \multicolumn{2}{|l|}{ PSITTACIFORMIS } \\
\hline \multicolumn{2}{|l|}{ Psittacidac } \\
\hline Loriculus bervllimus* & Sri Lankan lorikeet \\
\hline Psittacula kramerii & rose-ringed parakeet \\
\hline \multicolumn{2}{|l|}{ APOLHORMES } \\
\hline \multicolumn{2}{|l|}{ Apodidac } \\
\hline Cypsiurus balasinensis & palm switt \\
\hline \multicolumn{2}{|l|}{ STRIGIFORMES } \\
\hline \multicolumn{2}{|l|}{ Strigidae } \\
\hline Ninor scutulata & brown hawk owl \\
\hline \multicolumn{2}{|l|}{ COLUUMBIFORMES } \\
\hline \multicolumn{2}{|l|}{ Columbidac } \\
\hline Sreptopelia chinensis & spotted dove \\
\hline (halcophaps indica & emerald doc \\
\hline Treom pompadora & Pompadour green pigeon \\
\hline Ducula aenea & green imperial pigeon \\
\hline \multicolumn{2}{|l|}{ (IRUNFORMES } \\
\hline \multicolumn{2}{|l|}{ Rallidace } \\
\hline Amanromis phoenicurus & white-breasted walterhen \\
\hline \multicolumn{2}{|l|}{ CICONIFORMISS } \\
\hline \multicolumn{2}{|l|}{ Scolopacidac } \\
\hline (iallinago stenura" & pintail snipe \\
\hline Tringa hypolences ${ }^{\mathrm{m}}$ & common sandpiper \\
\hline \multicolumn{2}{|l|}{ Laridac } \\
\hline \multicolumn{2}{|l|}{ Stemidar Sternidar } \\
\hline Stema albifions & little tem \\
\hline \multicolumn{2}{|l|}{ Accipitridac } \\
\hline \multicolumn{2}{|l|}{ Accipitrinae } \\
\hline Haliastur indus & bralımini kite \\
\hline Haliacelus lewoogaster & white-bellied sea-cagle \\
\hline Spilornis cheela & crested serpent cagle \\
\hline Accipiter badins & shikra \\
\hline \multicolumn{2}{|l|}{ Haloerocoracidae } \\
\hline l'halocrocorax niger & little cormorant \\
\hline \multicolumn{2}{|l|}{ Ardeidae } \\
\hline Egretta garzetta & little egret \\
\hline Mesophoyax intermedia & median egret \\
\hline Ardeola graviilndian & pond heron \\
\hline Bubulcus ibis & cattle egret \\
\hline \multicolumn{2}{|l|}{ PASSERIFORMES } \\
\hline \multicolumn{2}{|l|}{ Irenidac } \\
\hline (hloropsis cochinchinensis & Jerdon's chloropsis \\
\hline Oriolidae/Campephagidae & \\
\hline ()riolini chinensis ${ }^{\text {in }}$ & black-naped oriole \\
\hline
\end{tabular}


Pericrecrotus cimmamomens Pericrocrotus flammens Dienurini Dicruridac

Dierurus macrocercus

Dicrmus caerulescens

Monarchini Monarchidae

Hypothymis azurea

Terpsiphone paradisi ${ }^{\text {inr }}$

Agithininac Chloropseidac

Acgithinia tiphia

Muscicapidac

Muscicapinae Muscicapidac

(Muscicapini)

M/uscicapa daumrica"'

ryomis trickelliase

Culicicape ceplonensis

Saxicolini

Saricoloides fillicata

Sturnidac

Gracula religiosa

Pycnonotidac

Picnomotus cafer

Hypsipetes lencocephalus

Zosteropidae

Zosterops palpebrosa

Sylviidac

Sylviinae

Timaliini Timaliidae

Nectrareniidac

(Nectareniinac)

Dicaeimi Dicacidac

Dicaenm erylhrorhynchos

Nectariini

Nectariinia zeylonica

Nectarinia asiatica

Passeridac

Motacillinac Motacillidac

Anthus nutulus

Estrildinac Estrildidae

Lonchura striata

Lonchura punctulat:a litle minivet

orange minivet

black drongo

white-vented drongo

azure flycatcher

paradise llycatcher

common iora

brown flycatcher

orange-breasted blue llycatcher

gray-headed thycatcher

black robin

common grackel

red-vented bulbul

black bulbul

small white-eye

common babbler

small tlower pecker

purple-rumped sunbird

purple sunbird

Indian pipit

white-backed munia

spotled mumia 